\title{
AM-Band: An Asymmetrical Multi-Band Model for Arterial Traffic Signal Coordination
}

\author{
Chao Zhang a, Yuanchang Xie ${ }^{\text {b, }}$, Nathan H. Gartner ${ }^{\text {b }}$, Chronis Stamatiadis ${ }^{\text {b }}$, and Tugba Arsava ${ }^{\text {b }}$ \\ ${ }^{a}$ Department of Civil and Environmental Engineering, Massachusetts Institute of Technology, Cambridge, MA \\ 02139, USA \\ ${ }^{b}$ Department of Civil and Environmental Engineering, University of Massachusetts Lowell, Lowell, MA 01854, USA
}

\begin{abstract}
MAXBAND and MULTIBAND are two well-recognized methods for arterial signal control. In this paper, an Asymmetrical Multi-BAND (AM-BAND) model is developed by relaxing the symmetrical progression band requirement in MULTIBAND. Such a relaxation allows the AM-BAND model to better utilize the available green times in each direction and to provide additional opportunities for vehicular progression. Similar to MULTIBAND, the proposed AM-BAND model is formulated as a mixed-integer linear program. However, in AM-BAND the green bands in each directional section of the arterials do not have to be symmetrical with respect to the progression line. The performance of the AM-BAND model is evaluated for two arterial network data using the AIMSUN microscopic traffic simulation tool. The optimal signal coordination plans are computed by the IBM CPLEX Optimization Studio and compared with signal timing plans generated by AM-BAND, MULTIBAND, and MAXBAND. Simulation results indicate that the traffic signal coordination plans generated by AM-BAND can provide significant benefits compared to those generated by MAXBAND and MULTIBAND.
\end{abstract}

\section{Keywords}

Traffic signal coordination, MAXBAND, MULTIBAND, CPLEX, mixed-integer linear programming, traffic simulation, AIMSUN

\section{Introduction}

Delay and number of stops are two main criteria often considered by traffic engineers for designing optimal arterial traffic signal control plans. To minimize delay and number of stops and to improve traffic operations efficiency, closely spaced traffic signals along an arterial are typically coordinated with a common cycle length and appropriate offsets such that a platoon of vehicles can travel along the entire arterial without stopping. The portion of the cycle time that allows a platoon of vehicles to traverse the arterial without stopping is termed the progression band for that direction. The width of the band is called bandwidth. By maximizing the inbound and outbound bandwidths along an arterial, vehicular progression along the arterial can be maintained and the total delay and number of stops can usually be minimized.

Other than minimizing delay and number of stops, properly coordinated traffic signals can effectively increase travel speed along the arterial, mitigate traffic emissions, and reduce the probabilities for rear-end collisions. Due to its significant impact on arterial traffic control and

* Corresponding author. Tel.: +1 9789343681; fax: +1 9789343052.

E-mail address: yuanchang_xie@uml.edu (Y. Xie). 
management, traffic signal coordination has long been a popular research area in traffic engineering. Research on arterial traffic signal coordination can generally be divided into two categories: one is maximizing the bandwidth along an arterial; the other is minimizing the total delay for the signalized system. Bandwidth-based models perform best when the arterial through traffic volume is dominant compared with the turning volume and the cross street volume. Delay-based models are usually difficult to solve due to their complexity and high degree of nonlinearity. Because of this, heuristic approaches are frequently used. Delay-based models have been implemented in some commercial traffic signal timing packages using macroscopic traffic simulation. Also, Park et al. (2001) proposed a delay-based method using genetic algorithm and microscopic traffic simulation. Despite the popularity of delay-based models, it was pointed out in Garter et al. (1991) that many traffic agencies find it important to impose upon the delay-based signal coordination solutions some arterial progression band constraints. This is critical for maintaining a smooth flow along the arterial. Due to this, some hybrid models integrating bandwidth maximization and delay minimization have been subsequently proposed (Cohen and Liu, 1986; Liu, 1988). Clearly, bandwidth maximization methods play an important role in the practice of traffic signal control and their continuous improvement and refinement is an important goal for the research community

In this paper, an improved version of a bandwidth-based arterial traffic signal coordination model, MULTIBAND (Gartner et al., 1990), is developed and tested. The formulation is based on mixed-integer linear programming and is solved by the IBM CPLEX ILOG Optimization Studio (IBM, 2013). This new version relaxes the symmetrical progression band requirement in MULTIBAND. More specifically, in this model the boundaries of the individual bands along each directional section of the arterial do not have to be symmetrical with respect to the progression line. Such a relaxation allows it to generate asymmetrical progression bands with varying widths for the different segments of the arterial. Compared to MULTIBAND, this model provides additional flexibility and can better utilize the available green times in each progression direction. The case studies presented in Section 5 demonstrate that this model can also provide significant benefits in performance.

\section{Literature review}

Brooks conducted one of the pioneer studies in arterial traffic signal coordination. He proposed a "half-integer algorithm" (Brooks, undated) to optimize the arterial progression bandwidth considering only two through phases. Messer et al. (1973) extended Brooks' half-integer algorithm to allow flexible selections of multiphase sequences. Several other researchers (Morgan and Little, 1964; Little, 1966; and Gartner et al., 1975) further advanced the work in this area by introducing a bandwidth maximization model based on mixed-integer linear programming. Later, Little et al. (1981) developed the MAXBAND model that maximizes the inbound and outbound bandwidths along an arterial via modifying cycle time, offsets and phase sequence patterns. Chang et al. (1988) extended the work by Little et al. (1981) and developed the MAXBAND-86 model. A major difference between MAXBAND-86 and MAXBAND is that MAXBAND-86 can solve multi-arterial closed network traffic signal coordination problems.

A fundamental limitation of MAXBAND and MAXBAND-86 is that they do not take into consideration the actual traffic volume and flow capacity of each link. Intuitively, it would be better for the signal control plan to be traffic dependent. In other words, the control plan should be related or proportional to the traffic volume and capacity of each directional link instead of 
being the same across the entire arterial. Without properly accounting for the link-specific traffic characteristics, the signal control plans that are generated may not provide optimal control performance due to the mismatch between demand and supply. Some segments may have excessive green times, while others may have inadequate green times that result in the frequent interruption of vehicle platoons.

To ensure that the signal coordination plan can suitably match the actual traffic demand, it is insufficient to use only the average ratio of inbound and outbound traffic volumes in the signal optimization model. The main reason is that different arterial segments may have quite different inbound and outbound traffic volume ratios. It is unlikely for them to equal the average ratio for the entire arterial. To address this issue with MAXBAND, Gartner et al. $(1990,1991)$ developed the MULTIBAND model which incorporates a set of systematic traffic-dependent criteria in the optimization objective. MULTIBAND is able to generate progression bands with varying widths using a mixed-integer linear programming model. It introduces a number of significant extensions based on MAXBAND and incorporates several important new features, including left-turn phase sequence optimization, initial queue clearance time, and variable bandwidths corresponding to each link's traffic characteristics. Simulation results demonstrated that MULTIBAND performs significantly better than MAXBAND.

Stamatiadis and Gartner (1996) extended the MULTIBAND model to arterial networks by creating MULTIBAND-96. With MULTIBAND-96, all the signal control variables in a network can be optimized. In the meantime, researchers at Texas Transportation Institute (TTI) (Chaudhary and Messer, 1993) developed an arterial traffic signal coordination program called "PASSER-IV", which is also designed to optimize traffic signal timings for both arterials and multi-arterial closed-loop networks based on the MAXBAND model.

In recent years, some other studies have been conducted on arterial traffic signal coordination with various levels of success. Given the abundance of literature in this area, it is challenging to provide an exhaustive review. Selected studies closely related to our research topic are briefly summarized below. Park et al. (1999; 2001) attempted to combine a genetic algorithm optimizer and mesoscopic/microscopic traffic simulation to find the optimal means to coordinate traffic signals. Lu et al. (2008) incorporated a traffic flow dispersion module in MAXBAND. Their goal was to address a limitation of the MAXBAND model which assumes that all vehicles travel at the same speed. Hu et al. (2011) pointed out that an assumption in the "half-integer algorithm" is invalid. They challenged the viewpoint that the lower and upper interferences cannot occur simultaneously at an intersection and proposed an improved algorithm. However, the solutions obtained from their new algorithm still need to be adjusted before implementation. Both MAXBAND and MULTIBAND models are considered un-split methods. In other words, the progress band in each direction is not split into two or more intervals within a cycle. Papola (1992) innovatively proposed an arterial traffic signal coordination model that can generate split solutions. It was found that the differences between the split and un-split solutions are marginal for arterials with sufficient number of traffic signals. Tian et al. (2008) assessed the impact of arterial left-turn phase sequence on arterial signal coordination. They found that lead-lag left-turn phase sequence is the most effective and the number of signals also has a significant impact on signal coordination effectiveness.

This study focuses on extending the MULTIBAND model by generating asymmetrical bands. In the next section, the MULTIBAND model is briefly presented first. Details on how to relax the symmetry requirement is then described in detail. 


\section{Methodology}

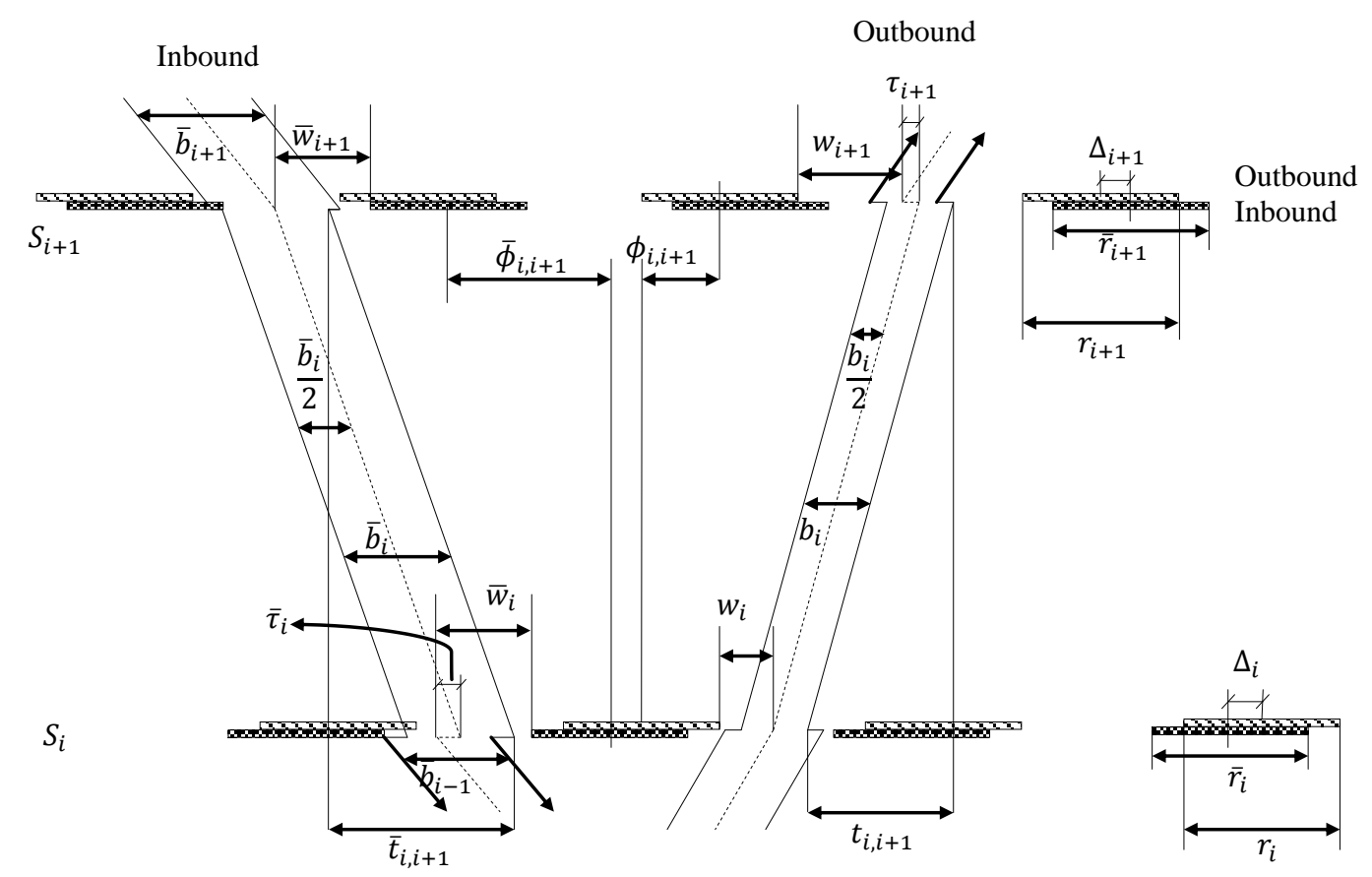

Fig. 1. Time-space diagram for MULTIBAND.

Both MULTIBAND and AM-BAND are modeled on a two-way arterial with $n$ intersections (nodes) using the symbols defined below. All intersections are controlled by a fixed-time strategy. As illustrated in Fig. 1, the two travel directions along the arterial are referred to as inbound and outbound, respectively. The traffic signal at each intersection $i$ is denoted by $S_{i}(i=1,2, \ldots, n)$. The following variables are defined:

$m_{i}=$ loop integer variable;

$b_{i}\left(\bar{b}_{i}\right)=$ outbound (inbound) bandwidth (cycles) for segment $i$;

$r_{i}\left(\bar{r}_{i}\right)=$ outbound (inbound) red time at $S_{i}$ (cycles);

$\tau_{i}\left(\bar{\tau}_{i}\right)=$ queue clearance time (cycles);

$w_{i}\left(\bar{w}_{i}\right)=$ interference variables (cycles);

$z=\frac{1}{C}=$ signal frequency (cycles/second);

$\phi_{i, i+1}\left[\bar{\phi}_{i, i+1}\right]=$ internode offsets (cycles);

$\Delta_{i}=$ intranode offset, which is the time difference between the center of $r_{i}$ and the nearest center of $\bar{r}_{i}$. It is positive if the center of $r_{i}$ is to the right of the center of $\bar{r}_{i}$;

$C_{1}, C_{2}=$ lower and upper limits on cycle length;

$k_{i}=$ target ratios of inbound to outbound bandwidth on section $i$;

$t_{i, i+1}\left[\bar{t}_{i, i+1}\right]=$ travel time from $S_{i}$ to $S_{i+1}$ outbound [S$S_{i+1}$ to $S_{i}$ inbound] (cycles);

$d_{i, i+1}\left[\bar{d}_{i, i+1}\right]=$ distance between $S_{i}$ and $S_{i+1}$ outbound [inbound] (feet);

$\delta_{i}\left(\bar{\delta}_{i}\right)=0 / 1$ variables through which the left turn pattern are determined;

$L_{i}\left(\bar{L}_{i}\right)=$ time allocated for outbound (inbound) left turn green at $S_{i}$ (cycles);

$G_{i}\left(\bar{G}_{i}\right)=$ outbound (inbound) green time for through traffic at $S_{i}$ (cycles); 
$e_{i}, f_{i}\left(\bar{e}_{i}, \bar{f}_{i}\right)=$ lower/upper limits on outbound (inbound) speed (feet/second); and $g_{i}, h_{i}\left(\bar{g}_{i}, \bar{h}_{i}\right)=$ lower/upper limits on change in outbound (inbound) speed (feet/second).

The interference variable $w_{i}$ refers to the time from the right side of red at $S_{i}$ to the progression line (i.e., the right dashed line in Fig. 1) of outbound green band, while $\bar{w}_{i}$ refers to the time from the left side of red at $S_{i}$ to the progression line (i.e., the left dashed line in Fig. 1) of inbound green band; queue clearance time refers to the time needed to clear the traffic turned onto main street from side streets; target ratio of inbound to outbound bandwidth $k_{i}$ is usually taken as the ratio of total inbound to total outbound traffic volumes for segment $i$; simplified notations are used for the travel time $t_{i, i+1}\left[\bar{t}_{i, i+1}\right]$ and distance $d_{i, i+1}\left[\bar{d}_{i, i+1}\right]$ variables: $t_{i}$ is used to represent $t_{i, i+1}$; the internode offset $\phi_{i, i+1}\left[\bar{\phi}_{i, i+1}\right]$ refers to the time from center of an outbound[inbound] red at $S_{i}$ to the center of the closest outbound[inbound] red at $S_{i+1}$. $\phi_{i, i+1}\left[\bar{\phi}_{i, i+1}\right]$ is positive if the center of the red at $S_{i+1}$ is to the right[left] of the current red at $S_{i}$.

By observing the time-space diagram in Figs. 1 and 3, we notice a loop which starts from the center of outbound red at $S_{i}$ and ends at the same point. This loop consists of the center of outbound red at $S_{i+1}$, the center of inbound red at $S_{i+1}$, and the center of inbound red at $S_{i}$. This can be mathematically described in the Eq. (1) below.

$$
\begin{array}{r}
\left(t_{i}+\bar{t}_{i}\right)+\frac{1}{2}\left(r_{i}+\bar{r}_{i}\right)+\left(w_{i}+\bar{w}_{i}\right)-\frac{1}{2}\left(r_{i+1}+\bar{r}_{i+1}\right)-\left(w_{i+1}+\bar{w}_{i+1}\right) \\
-\left(\tau_{i+1}+\bar{\tau}_{i}\right)+\left(\Delta_{i}-\Delta_{i+1}\right)=m_{i+1} \quad i=1, \ldots, n-1
\end{array}
$$

The so-called loop integer constraint is due to the fact that all signals along the arterial are operated with a common cycle time. This equation is also the basis for the derivation of Eqs. (7) and (23).

The MULTIBAND model can also optimize left-turn phase sequences. This is done by introducing two binary variables $\delta_{i}\left(\bar{\delta}_{i}\right)$. Different left-turn phase sequences can be represented by these two variables as shown in Table 1 and Fig. 2 . Table 1 shows that the intranode offset can also be calculated based on these two binary variables.

\section{Table 1}

Left-turn patterns.

\begin{tabular}{ccccc}
\hline Pattern & $\Delta_{i}$ & $\delta_{i}$ & $\bar{\delta}_{i}$ & Note \\
\hline 1 & $-0.5\left(L_{i}+\bar{L}_{i}\right)$ & 0 & 1 & Outbound left leads/inbound lags \\
2 & $0.5\left(L_{i}+\bar{L}_{i}\right)$ & 1 & 0 & Outbound left lags/inbound leads \\
3 & $-0.5\left(L_{i}-\bar{L}_{i}\right)$ & 0 & 0 & Outbound left leads/inbound leads \\
4 & $0.5\left(L_{i}-\bar{L}_{i}\right)$ & 1 & 1 & Outbound left lags/inbound lags \\
\hline
\end{tabular}


Outbound left leads/ inbound lags
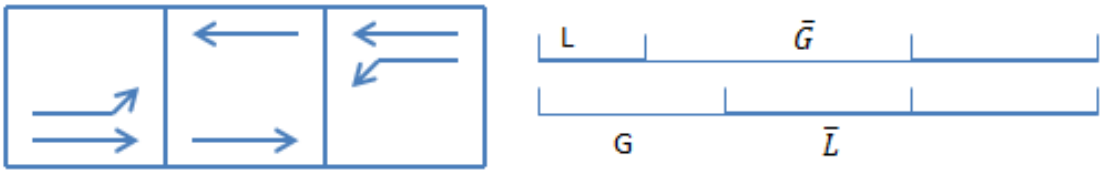

INBOUND

OUTBOUND

Outbound left lags/ inbound leads
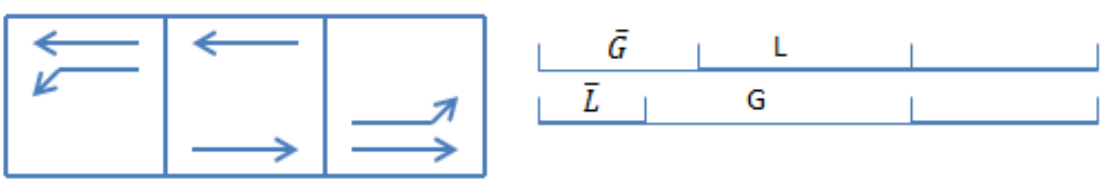

INBOUND

OUTBOUND

Outbound left leads/ inbound leads
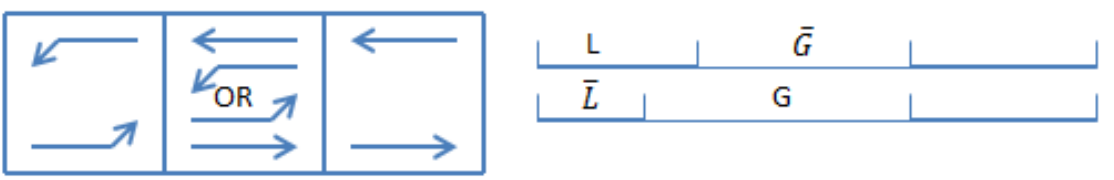

INBOUND

OUTBOUND

Outbound left lags/ inbound lags
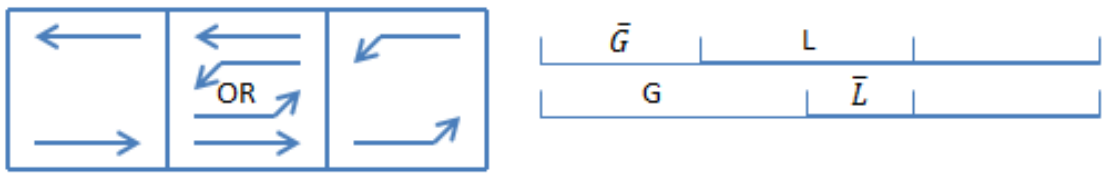

INBOUND

OUTBOUND

Fig. 2. The four possible patterns of left-turn phases.

\subsection{MULTIBAND}

In MAXBAND (Little et al., 1981), all road segments along each arterial direction have a uniform bandwidth. In MULTIBAND, bandwidths of different road segments are defined individually. The individual bandwidths are weighted based on their corresponding road segments' volume to saturation flow rate ratios. Assuming intersections are numbered sequentially from 1 to $n$ in the outbound direction, the MULTIBAND model is defined in Eqs. (2) (12). The decision variables for this model include $b_{i}, \bar{b}_{i}, z, w_{i}, \bar{w}_{i}, t_{i}, \bar{t}_{i}, \delta_{i}, \bar{\delta}_{i}, m_{i}$. These variables were defined above.

where,

$$
\operatorname{Max} B=\frac{1}{n-1} \sum_{i=1}^{n-1}\left(a_{i} b_{i}+\bar{a}_{i} \bar{b}_{i}\right)
$$

and

$$
a_{i}=\left(\frac{v_{i}}{s_{i}}\right)^{p} \quad \bar{a}_{i}=\left(\frac{\bar{v}_{i}}{\bar{s}_{i}}\right)^{p}
$$

$V_{i}\left(\bar{V}_{i}\right)=$ outbound (inbound) total directional volume on section $i$,

$S_{i}\left(\bar{S}_{i}\right)=$ outbound (inbound) saturation flow on section $i$, and

$p=$ exponential power; the values $p=0,1,2$, and 4 were used.

s.t.

$$
\left(1-k_{i}\right) \bar{b}_{i} \geq\left(1-k_{i}\right) k_{i} b_{i} \quad i=1, \ldots, n-1
$$




$$
\begin{aligned}
& 1 / C_{2} \leq z \leq 1 / C_{1} \\
& \left\{\begin{array}{c}
\frac{1}{2} b_{i} \leq w_{i} \leq\left(1-r_{i}\right)-\frac{1}{2} b_{i} \\
\frac{1}{2} b_{i} \leq w_{i+1}+\tau_{i+1} \leq\left(1-r_{i+1}\right)-\frac{1}{2} b_{i} \\
\frac{1}{2} \bar{b}_{i} \leq \bar{w}_{i}-\bar{\tau}_{i} \leq\left(1-\bar{r}_{i}\right)-\frac{1}{2} \bar{b}_{i}
\end{array} \quad i=1, \ldots, n-1\right. \\
& \frac{1}{2} \bar{b}_{i} \leq \bar{w}_{i}-\bar{\tau}_{i} \leq\left(1-\bar{r}_{i}\right)-\frac{1}{2} \bar{b}_{i} \\
& \frac{1}{2} \bar{b}_{i} \leq \bar{w}_{i+1} \leq\left(1-\bar{r}_{i+1}\right)-\frac{1}{2} \bar{b}_{i} \\
& \left(w_{i}+\bar{w}_{i}\right)-\left(w_{i+1}+\bar{w}_{i+1}\right)+\left(t_{i}+\bar{t}_{i}\right)+\delta_{i} L_{i}-\bar{\delta}_{i} \bar{L}_{i}-\delta_{i+1} L_{i+1}+\bar{\delta}_{i+1} \bar{L}_{i+1}-m_{i}= \\
& \left(r_{i+1}-r_{i}\right)+\left(\tau_{i+1}+\bar{\tau}_{i}\right) \quad i=1, \ldots, n-1 \\
& \left\{\left(d_{i} / f_{i}\right) z \leq t_{i} \leq\left(d_{i} / e_{i}\right) z\right. \\
& \left\{\left(\bar{d}_{i} / \bar{f}_{i}\right) z \leq \bar{t}_{i} \leq\left(\bar{d}_{i} / \bar{e}_{i}\right) z\right. \\
& i=1, \ldots, n-1 \\
& \left\{\begin{array}{l}
\left(d_{i} / h_{i}\right) z \leq\left(d_{i} / d_{i+1}\right) t_{i+1}-t_{i} \leq\left(d_{i} / g_{i}\right) z \\
\left(\bar{d}_{i} / \bar{h}_{i}\right) z \leq\left(\bar{d}_{i} / \bar{d}_{i+1}\right) \bar{t}_{i+1}-\bar{t}_{i} \leq\left(\bar{d}_{i} / \bar{g}_{i}\right) z
\end{array}\right. \\
& \left(\bar{d}_{i} / \bar{h}_{i}\right) z \leq\left(\bar{d}_{i} / \bar{d}_{i+1}\right) \bar{t}_{i+1}-\bar{t}_{i} \leq\left(\bar{d}_{i} / \bar{g}_{i}\right) z \quad i=1, \ldots, n-2 \\
& b, \bar{b}, z, w_{i}, \bar{w}_{i}, t_{i}, \bar{t}_{i} \geq 0 \\
& m_{i} \text { integer } \\
& \delta_{i}, \bar{\delta}_{i} \text { zero/one variables }
\end{aligned}
$$

Constraint (4) allows the arterial direction with a larger traffic volume to have a wider progression band; constraint (5) defines the lower and upper limits of cycle length. Two reciprocals are used here so that the remaining constraints can be expressed in linear form; (6) is an interference constraint. It ensures that the left and right boundaries of a band do not interfere with the right and left edges of red signals, respectively. As shown in Fig. 1, $w_{i}$ is the time from the right edge of red time at $S_{i}$ to the progression line of outbound progression band. $\bar{w}_{i}$ is defined similarly; constraint (7) is the well-known loop integer constraint (see Gartner et al., 1991). One difference is that in MULTIBAND Gartner et al. (1991) changed the reference line used for defining the interference variables $w_{i}\left(\bar{w}_{i}\right)$. Also, they included four additional variables $\delta_{i}, \bar{\delta}_{i}, L_{i}, \bar{L}_{i}$. In this way, the left-turn phase sequence can also be optimized; constraints (9) and (10) impose upper and lower limits on the travel speed of each segment and the speed change between consecutive segments.

MULTIBAND provides considerable flexibilities compared with MAXBAND. Instead of generating a uniform bandwidth for each travel direction, it generates variable bandwidths for each segment of the arterial based on its corresponding volume to saturation flow rate ratio. However, a requirement in MULTIBAND is that the interference constraints are formulated based on the centerlines (see the dashed lines in Fig. 1) of the progression bands. In other words, the progression lines are used as the reference lines to derive the interference constraints and the progression band in each direction is symmetrical along the progression line. This restriction can prevent it from fully utilizing the available green times on both sides of the progression line. To address this issue, AM-BAND relaxes this restriction by generating asymmetrical bandwidths along the arterial. To maintain progression, there is still a continuous progression line in each direction; however, the bandwidths for each directional segment along the arterial need not be symmetrical anymore. This relaxation provides additional flexibility to the original MULTIBAND model and enhances its performance.

\subsection{AM-BAND}




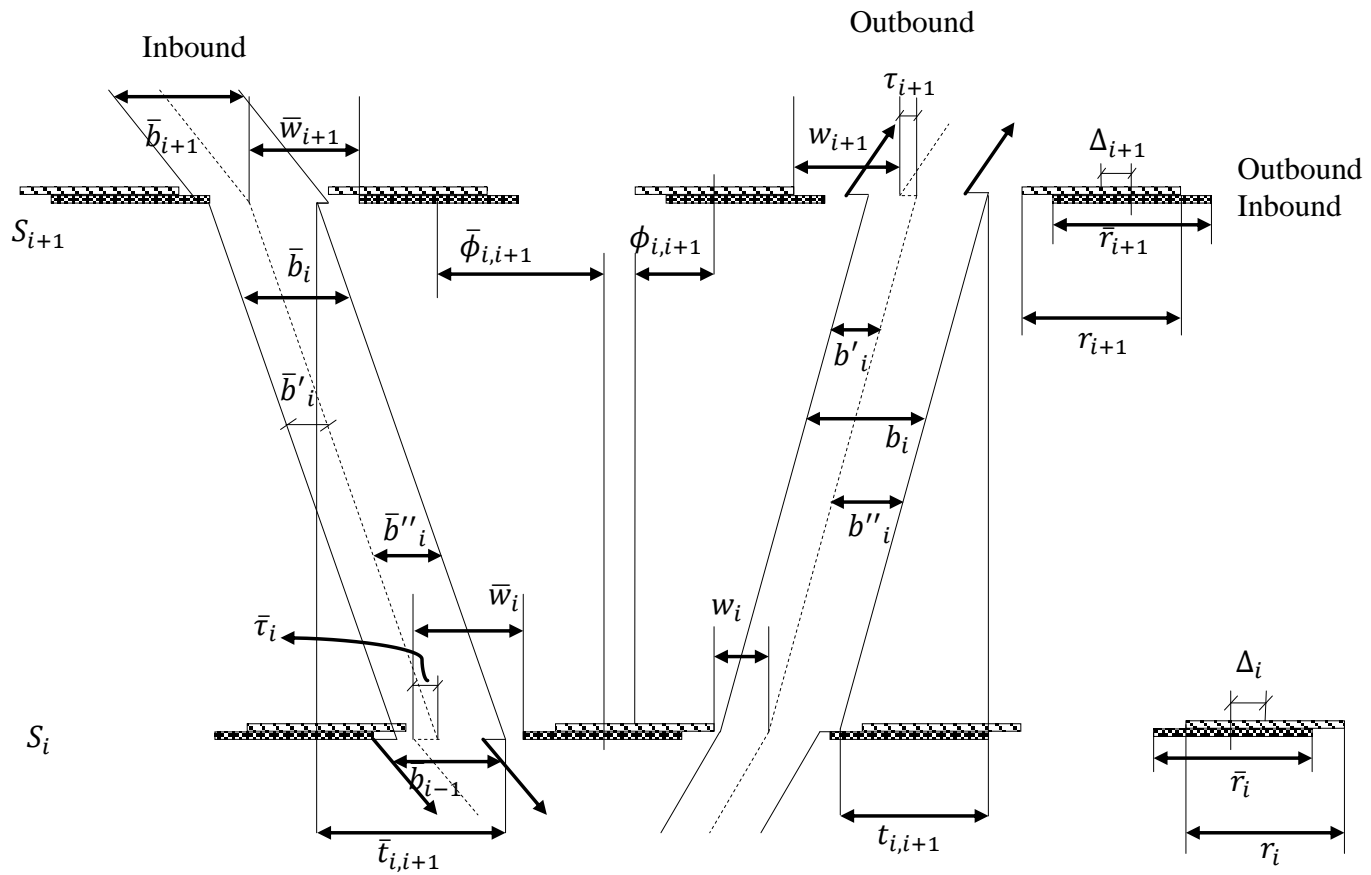

Fig. 3. Time-space diagram for AM-BAND.

In AM-BAND, the progression band for each directional road section (both inbound and outbound), $b_{i}$ and $\bar{b}_{i}$, in MULTIBAND is separated into two components in (13), represented by $b^{\prime}{ }_{i}$ and $b^{\prime \prime}{ }_{i}$ for outbound and $\bar{b}^{\prime}{ }_{i}$ and $\bar{b}^{\prime \prime}{ }_{i}$ for inbound. The left (e.g., $b^{\prime}{ }_{i}$ ) and right (e.g., $b^{\prime \prime}{ }_{i}$ ) components do not need to be equal as shown in Fig. 3. In other words, the progression bands in AM-BAND do not need to be symmetrical along the reference lines (i.e., progression lines in MULTIBAND) used to derive the interference constraint. Therefore, we have:

$$
\left\{\begin{array}{l}
b_{i} \rightarrow b_{i}^{\prime}+b^{\prime \prime}{ }_{i} \\
\bar{b}_{i} \rightarrow \bar{b}_{i}^{\prime}+\bar{b}^{\prime \prime}{ }_{i}
\end{array}\right.
$$

Based on the above revised notations for the progression bands, corresponding changes are also made to the interference constraints. The interference constraint (6) in MULTIBAND for outbound intersection $S_{i}$ now becomes:

$$
\left\{\begin{array}{c}
w_{i}+b^{\prime \prime}{ }_{i} \leq 1-r_{i} \\
w_{i}-b^{\prime}{ }_{i} \geq 0
\end{array}\right.
$$

where $w_{i}$ is defined in the same way as in MULTIBAND. By combining both inequalities in (14), the following new interference constraint is obtained for intersections $S_{i}$ and $S_{i+1}$ :

$$
\left\{\begin{array}{c}
b^{\prime}{ }_{i} \leq w_{i} \leq\left(1-r_{i}\right)-b^{\prime \prime}{ }_{i} \\
b_{i}^{\prime} \leq w_{i+1}+\tau_{i+1} \leq\left(1-r_{i+1}\right)-b^{\prime \prime}{ }_{i}
\end{array}\right.
$$


Similarly, another set of interference constraints for $\bar{w}_{i}$ and $\bar{w}_{i+1}$ is obtained for the inbound direction as shown below in (16).

$$
\left\{\begin{array}{l}
\bar{b}^{\prime \prime}{ }_{i} \leq \bar{w}_{i}-\bar{\tau}_{i} \leq\left(1-\bar{r}_{i}\right)-\bar{b}^{\prime}{ }_{i} \\
\bar{b}^{\prime \prime}{ }_{i} \leq \bar{w}_{i+1} \leq\left(1-\bar{r}_{i+1}\right)-\bar{b}^{\prime}{ }_{i}
\end{array}\right.
$$

To ensure that each "left" or "right" part of a progression band is non-zero, the following constraint is added to the AM-BAND model:

$$
\left\{\begin{array}{l}
(1 / q) \cdot b^{\prime \prime}{ }_{i} \leq b^{\prime}{ }_{i} \leq q \cdot b^{\prime \prime}{ }_{i} \\
(1 / q) \cdot \bar{b}^{\prime \prime}{ }_{i} \leq \bar{b}^{\prime}{ }_{i} \leq q \cdot \bar{b}^{\prime \prime}{ }_{i}
\end{array}\right.
$$

where $q$ can be any positive real number (e.g., 1, 2, 3, ..). In this study, $q$ is set to 2 based on an empirical sensitivity study. The complete AM-BAND model is presented below. The objective function is similar to that of the MULTIBAND model. The main difference is that $b_{i}$ and $\bar{b}_{i}$ are now replaced by $b^{\prime}{ }_{i}+b^{\prime \prime}{ }_{i}$ and $\bar{b}^{\prime}{ }_{i}+\bar{b}^{\prime \prime}{ }_{i}$, respectively. The optimization program determines the following decision variables: $b^{\prime}{ }_{i}, b^{\prime \prime}{ }_{i}, \bar{b}^{\prime}{ }_{i}, \bar{b}^{\prime \prime}{ }_{i}, z, w_{i}, \bar{w}_{i}, t_{i}, \bar{t}_{i}, \delta_{i}, \bar{\delta}_{i}, m_{i}$.

$$
\operatorname{Max} B=\frac{1}{n-1} \sum_{i=1}^{n-1}\left[a_{i}\left(b_{i}^{\prime}+b^{\prime \prime}{ }_{i}\right)+\bar{a}_{i}\left(\bar{b}_{i}^{\prime}+\bar{b}^{\prime \prime}{ }_{i}\right)\right]
$$

s.t.

$$
\begin{aligned}
& \left(1-k_{i}\right)\left(\bar{b}_{i}^{\prime}+\bar{b}^{\prime \prime}{ }_{i}\right) \geq\left(1-k_{i}\right) k_{i}\left(b^{\prime}{ }_{i}+b^{\prime \prime}{ }_{i}\right) \quad i=1, \ldots, n-1 \\
& 1 / C_{2} \leq z \leq 1 / C_{1} \\
& \left\{\begin{array}{c}
b^{\prime}{ }_{i} \leq w_{i} \leq\left(1-r_{i}\right)-b^{\prime \prime}{ }_{i} \\
b^{\prime}{ }_{i} \leq w_{i+1}+\tau_{i+1} \leq\left(1-r_{i+1}\right)-b^{\prime \prime}{ }_{i} \\
\bar{b}^{\prime \prime}{ }_{i} \leq \bar{w}_{i}-\bar{\tau}_{i} \leq\left(1-\bar{r}_{i}\right)-\bar{b}^{\prime}{ }_{i}
\end{array} \quad i=1, \ldots, n-1\right. \\
& \bar{b}^{\prime \prime}{ }_{i} \leq \bar{w}_{i+1} \leq\left(1-\bar{r}_{i+1}\right)-\bar{b}^{\prime}{ }_{i} \\
& \left\{\begin{array}{l}
(1 / 2) \cdot b^{\prime \prime}{ }_{i} \leq b^{\prime}{ }_{i} \leq 2 \cdot b^{\prime \prime}{ }_{i} \\
(1 / 2) \cdot \bar{b}^{\prime \prime}{ }_{i} \leq \bar{b}^{\prime}{ }_{i} \leq 2 \cdot \bar{b}^{\prime \prime}{ }_{i}
\end{array} \quad i=1, \ldots, n-1\right. \\
& \left(w_{i}+\bar{w}_{i}\right)-\left(w_{i+1}+\bar{w}_{i+1}\right)+\left(t_{i}+\bar{t}_{i}\right)+\delta_{i} L_{i}-\bar{\delta}_{i} \bar{L}_{i}-\delta_{i+1} L_{i+1}+\bar{\delta}_{i+1} \bar{L}_{i+1}-m_{i}= \\
& \left(r_{i+1}-r_{i}\right)+\left(\tau_{i+1}+\bar{\tau}_{i}\right) \quad i=1, \ldots, n-1 \\
& \left\{\begin{array}{l}
\left(d_{i} / f_{i}\right) z \leq t_{i} \leq\left(d_{i} / e_{i}\right) z \\
\left(\bar{d}_{i} / \bar{f}_{i}\right) z \leq t_{i} \leq\left(\bar{d}_{i} / \bar{e}_{i}\right) z
\end{array} \quad \boldsymbol{i}=\mathbf{1}, \ldots, n-1\right. \\
& \left\{\begin{array}{l}
\left(d_{i} / h_{i}\right) z \leq\left(d_{i} / d_{i+1}\right) t_{i+1}-t_{i} \leq\left(d_{i} / g_{i}\right) z \\
\left(\bar{d}_{i} / \bar{h}_{i}\right) z \leq\left(\bar{d}_{i} / \bar{d}_{i+1}\right) \bar{t}_{i+1}-\bar{t}_{i} \leq\left(\bar{d}_{i} / \bar{g}_{i}\right) z
\end{array} \quad i=1, \ldots, n-2\right. \\
& b^{\prime}{ }_{i}, b^{\prime \prime}{ }_{i}, \bar{b}^{\prime}{ }_{i}, \bar{b}^{\prime \prime}{ }_{i}, z, w_{i}, \bar{w}_{i}, t_{i}, \bar{t}_{i} \geq 0 \\
& m_{i} \text { integer } \\
& \delta_{i}, \bar{\delta}_{i} \text { zero/one variables }
\end{aligned}
$$

In MULTIBAND the centerlines of the progression bands (also called "the progression lines") are indicated by the dashed lines in Fig. 1. These progression lines are used as the references to derive the interference constraints in (6) and the loop integer constraints in (7). As shown in (6), a progression band is strictly symmetrical with respect to its corresponding progression line. The 
locations of these progression lines are not predefined or predetermined by the analyst. They are determined as a result of the MULTIBAND optimization model. Once the values of $b_{i}, \bar{b}_{i}, z, w_{i}, \bar{w}_{i}, t_{i}$ and $\bar{t}_{i}$ are optimized, one can determine the exact locations of the progression lines. In AM-BAND, there are also progression lines (the dashed lines in Fig. 3) to ensure the continuity of the link-specific bands in each direction of the arterial. In addition, they are used as references to derive the interference constraints in (21) and the loop integer constraints in (23). The two components of each band $\left(b^{\prime}{ }_{i}\right.$ and $\left.b^{\prime \prime}{ }_{i}\right)$, however, are no longer required to be equal (i.e., symmetrical with respect to the corresponding progression line) as shown in (21). Therefore, in AM-BAND the progression lines are not the centers of the progression bands. These progression lines are not predefined or predetermined either. Similarly, once $b^{\prime}{ }_{i}, b^{\prime \prime}{ }_{i}, \bar{b}^{\prime}{ }_{i}, \bar{b}^{\prime \prime}{ }_{i}, z, w_{i}, \bar{w}_{i}, t_{i}$ and $\bar{t}_{i}$ are optimized, the locations of the progression lines can be readily determined.

As shown in Fig. 1, the left and right boundaries of a progression band generated by MULTIBAND have to be symmetrical with respect to the progression line. This requirement restricts the left and right boundaries from further expanding due to interferences on either side of the progression band. For instance, there might be available green time for the right boundary to further expand. However, due to an interference on the left boundary, one cannot expand the right boundary as much as possible. The AM-BAND model relaxes this symmetry restriction by introducing two separate variables $b^{\prime}{ }_{i}$ and $b^{\prime \prime}{ }_{i}$ (using the outbound direction as an example). In the case of the previous example, the right boundary of the progression band can be expanded regardless of the interference on the left boundary. This relaxation allows the progression bands to fully utilize all available green times. These additional bandwidths can help to dissipate the overflow queues from previous cycles as well as accommodate in/out traffic from the cross streets. Table 2 compares the number of constraints and variables for MAXBAND, MULTIBAND, and AM-BAND. Although AM-BAND increases substantially the number of variables and constraints, this causes no computational difficulty with present day computers.

\section{Test design \& data collection}

Data from two arterials are used to evaluate the AM-BAND model and compare it with MAXBAND and MULTIBAND. These two arterials are shown in Figs. 3 and 4, respectively. In both figures, the numbers in parentheses represent traffic information. For example, $(120,366,0)$ means the total link volume is 486 vehicles per hour. Among them, there are 120 left-turn vehicles, 366 through vehicles, and 0 right-turn vehicles. The $k$ values on the left side of both figures are the ratios of the inbound to outbound volumes for the corresponding sections. Distances between consecutive intersections are also provided. For each arterial, all intersections are controlled by the pre-timed strategy and share the same cycle length. 
Table 2

Constraints and variables for AM-BAND compared to MAXBAND and MULTIBAND.

\begin{tabular}{|c|c|c|c|c|c|c|c|}
\hline \multicolumn{2}{|c|}{ Type } & \multicolumn{2}{|c|}{ MAXBAND } & \multicolumn{2}{|c|}{ MULTIBAND } & \multicolumn{2}{|c|}{ AM-BAND } \\
\hline Constraint & Variable & $\begin{array}{c}\text { No. of } \\
\text { Constraints }\end{array}$ & $\begin{array}{c}\text { No. of } \\
\text { Variables }\end{array}$ & $\begin{array}{c}\text { No. of } \\
\text { Constraints }\end{array}$ & $\begin{array}{c}\text { No. of } \\
\text { Variables }\end{array}$ & $\begin{array}{c}\text { No. of } \\
\text { Constraints }\end{array}$ & $\begin{array}{c}\text { No. of } \\
\text { Variables }\end{array}$ \\
\hline Band & $b^{\prime}{ }_{i}, b^{\prime \prime}{ }_{i}, \bar{b}_{i}^{\prime}, \bar{b}^{\prime \prime}{ }_{i}$ & - & 2 & - & $2(n-1)$ & - & $4(n-1)$ \\
\hline Bandwidth & $k_{i}$ & 1 & - & $\mathrm{n}-1$ & - & $2(n-1)$ & - \\
\hline Cyclic Rate & $\mathrm{z}$ & 2 & 1 & 2 & 1 & 2 & 1 \\
\hline Interference & $w_{i}, \bar{w}_{i}$ & $2 n$ & $2 n$ & $8(n-1)$ & $2 n$ & $8(n-1)$ & $2 n$ \\
\hline Loop Integer & $m_{i}$ & $\mathrm{n}-1$ & $\mathrm{n}-1$ & $\mathrm{n}-1$ & $\mathrm{n}-1$ & $\mathrm{n}-1$ & $\mathrm{n}-1$ \\
\hline Travel Time & $t_{i}, \bar{t}_{i}$ & $4(n-1)$ & $2(n-1)$ & $4(n-1)$ & $2(n-1)$ & $4(n-1)$ & $2(n-1)$ \\
\hline Speed Change & - & $4(n-2)$ & - & $4(n-2)$ & - & $4(n-2)$ & - \\
\hline Left-Right Band Size & - & - & - & - & - & $4(n-1)$ & - \\
\hline Total & & $11 \mathrm{n}-10$ & $5 n$ & $18 n-20$ & $7 n-4$ & $23 n-21$ & $9 n-6$ \\
\hline \multirow{2}{*}{ Example } & $\mathrm{n}=5$ & 45 & 25 & $70(+55 \%)$ & $31(+24 \%)$ & $94(+109 \%)$ & $39(+56 \%)$ \\
\hline & $\mathrm{n}=10$ & 100 & 50 & $160(+60 \%)$ & $66(+32 \%)$ & $209(+109 \%)$ & $84(+68 \%)$ \\
\hline
\end{tabular}




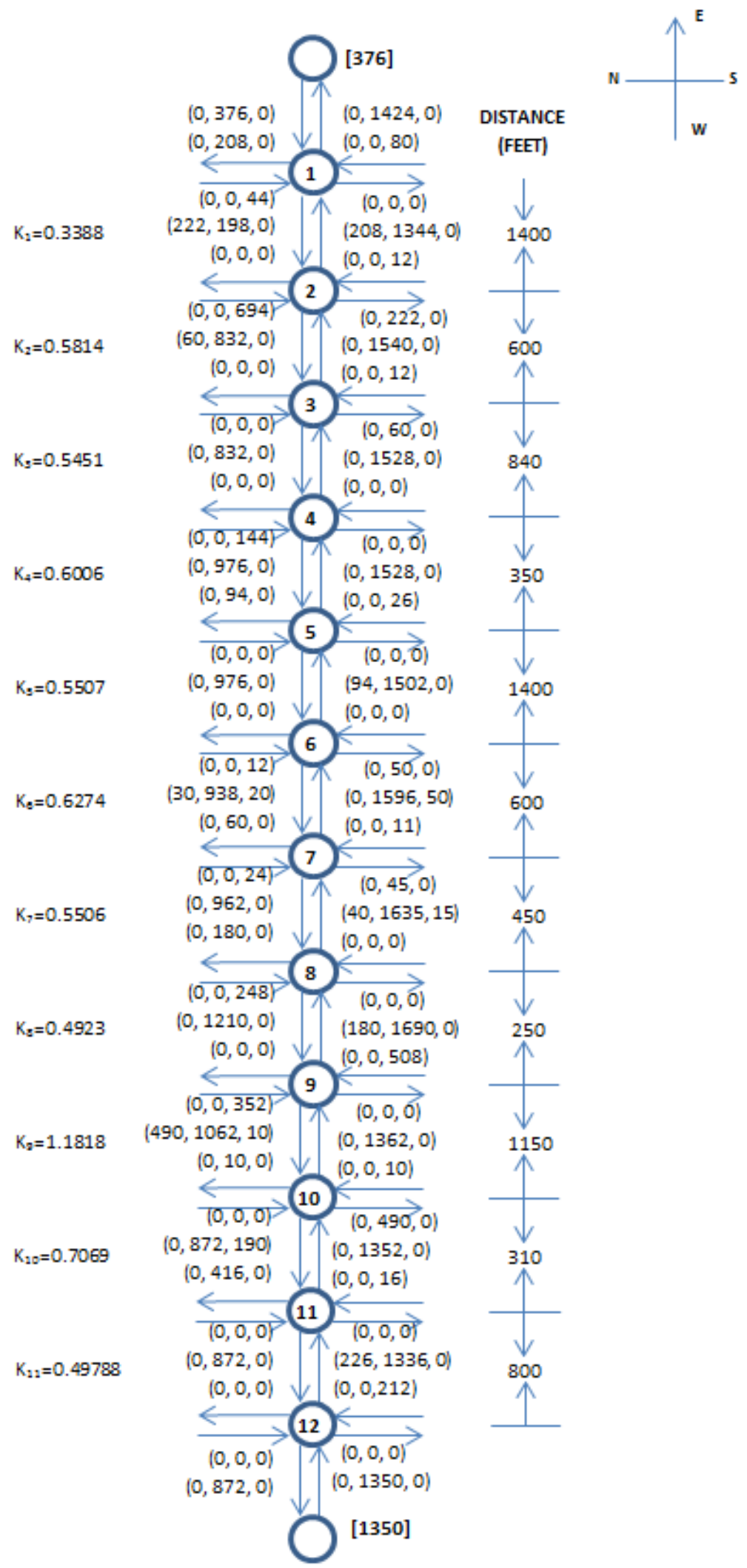

Fig. 4. Data for Case I Arterial. 
$K_{1}=4.2148$

$K_{2}=4.0741$

$\mathrm{Kg}=2.5786$

$K_{4}=1.4800$

$\mathrm{K}_{\mathrm{g}}=1.5304$

$K_{A}=1.2818$

$K_{7}=1.2818$

$K_{s}=0.6888$

$K_{2}=0.5317$

$K_{10}=0.3796$

$K_{11}=0.1577$

$K_{22}=0.1141$

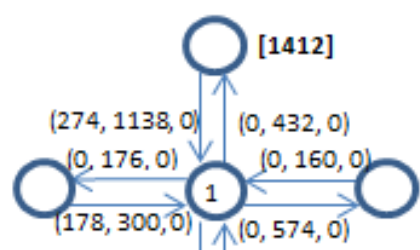

$(38,1100,0) \quad(16,254,0)$

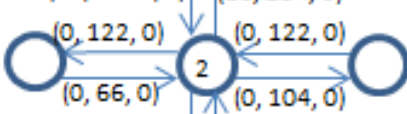

$(280,820,0) \quad(0,270,0)$

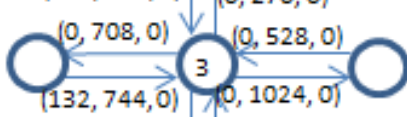

$(154,666,0) \quad(180,138,0)$

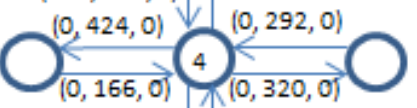

$(112,554,0) \quad(132,318,0)$

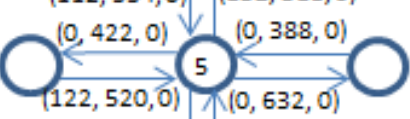

$(90,464,0) \quad(34,328,0)$

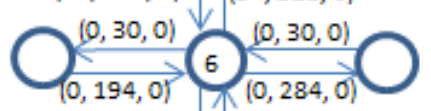

$(0,464,0) \quad(0,362,0)$

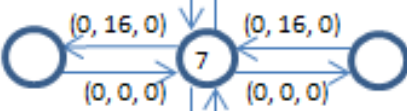

$(132,332,0) \quad(0,362,0)$

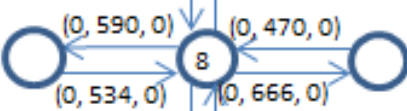

$(40,292,0) \quad(120,362,0)$

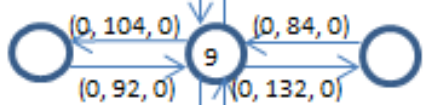

$(84,208,0) \quad(20,482,0)$

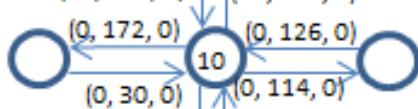

$(114,94,0) \quad(46,502,0)$

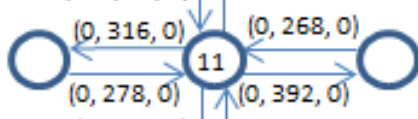

$(26,68,0) \quad(48,548,0)$

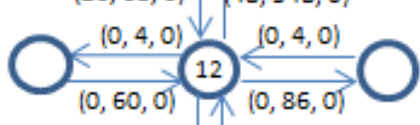

$(68,0,0) \quad(0,596,0)$

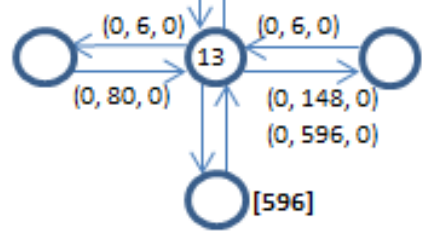

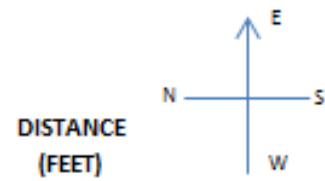

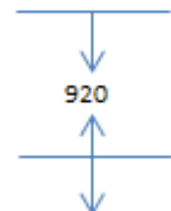

2600
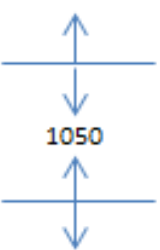

1120

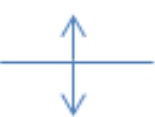

830
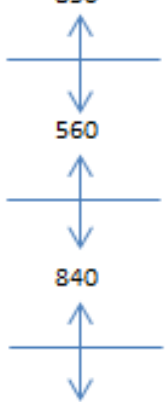

1370
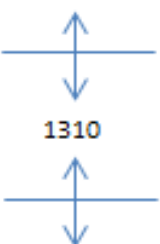

1280

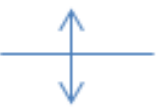

1050

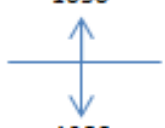

1280

个

Fig. 5. Data for Case II Arterial. 
In the interest of brevity, the two arterials are referred to as Case I and Case II, respectively. In each case, a total of nine scenarios are considered as shown in Table 3, where $p$ is an exponential power that directly affects the weights for individual progression bandwidths in (2) and (18). Each scenario is coded in AIMSUN (TSS, 2013) and simulated for 100 replications. The mean and standard deviation of each measure of effectiveness (MOE) are calculated. MOEs include average delay time, density, average stop time, average speed, harmonic speed and average number of stops.

Table 3

Different simulation cases and scenarios.

\begin{tabular}{|c|c|c|}
\hline \multirow{2}{*}{ Model } & \multicolumn{2}{|c|}{ Number of Scenarios } \\
\hline & Case I & Case II \\
\hline MAXBAND & 1 & 1 \\
\hline MULTIBAND & $4(p=0, p=1, p=2, p=4)$ & $4(p=0, p=1, p=2, p=4)$ \\
\hline AM-BAND & $4(p=0, p=1, p=2, p=4)$ & $4(p=0, p=1, p=2, p=4)$ \\
\hline
\end{tabular}

\section{Results analysis}

Given input data such as red times, upper/lower cycle time limits, and distance between two adjacent signals, CPLEX (IBM, 2013) is used to generate the optimal signal coordination plans for the two arterials using the MAXBAND, MULTIBAND, and AM-BAND models. The signal control parameters optimized by CPLEX include bandwidth, left-turn pattern, travel time/speed, interference value, and cycle length. The optimized traffic signal control plans are evaluated using the AIMSUN simulation model and the results are presented below. A desktop computer is used in this study. It has an Intel i-7 CPU, 8 GB RAM and runs a 64-bit Windows 7 operating system. For each MILP model, CPLEX is able to obtain the optimal solution in less than one minute.

\subsection{AIMSUN simulation results}

The simulation results for Cases I and II are summarized in Tables 4 and 5, respectively. In AIMSUN, the delay time refers to the difference between the expected travel time and the actual travel time. Here the expected travel time is defined as the time it would take to traverse the arterial under ideal conditions. The average delay in both Tables 4 and 5 is calculated as the average of the delay time of each vehicle and has a unit of $\mathrm{sec} / \mathrm{veh} / \mathrm{mi}$. The stop time is calculated similarly and has the same unit as the average delay.

According to Tables 4 and 5, the performance of AM-BAND is better than MAXBAND in terms of all MOEs for all $p$ values. The standard deviations are small enough compared to the means, which suggests the results are statistically significant. For MULTIBAND and AM-BAND, the results vary for different $p$ values. However, the performance differences within each method are much smaller than between the methods. The overall performance of AM-BAND is better than MULTIBAND. Also, the results suggest that there is no clear indication which weighting option (i.e., $p$ value) is superior. This is an area which will require further studies.

To better illustrate the relative improvements brought by AM-BAND compared to MAXBAND and compared to MULTIBAND, the percentage improvements in terms of average 
delay and average number of stops are shown in Tables 6 and 7. The results show clearly that AM-BAND outperforms both MAXBAND and MULTIBAND. In Case I, AM-BAND can reduce delay by up to $34.00 \%$ compared with MAXBAND and by up to $9.04 \%$ compared with MULTIBAND. In Case II, the corresponding reductions are up to $23.73 \%$ and up to $8.52 \%$, respectively. Reductions in average number of stops in Case I are up to $10.53 \%$ compared with MAXBAND and up to 5.36\% compared with MULTIBAND. For Case II, the corresponding reductions are up to $21.37 \%$ and $11.54 \%$, respectively. In the practice of traffic engineering, such improvements are very significant, especially considering that MULTIBAND has already provided improvements of approximately $13-28 \%$ in average delay and $2-11 \%$ in average number of stops over MAXBAND.

The distributions of all MOEs are also calculated for both cases. Due to the page limit, only the average delay and average speed results for Case I are shown in Fig. 6. The red bars show the 95\% confidence intervals and the crosses represent the corresponding mean MOE values. From Fig. 6, one can see that both AM-BAND and MULTIBAND clearly outperform MAXBAND. The advantage of AM-BAND over MULTIBAND is relatively less significant. 
Table 4

Simulation results for Case I.

\begin{tabular}{ccccccc}
\hline Method & $\begin{array}{c}\text { Average Delay } \\
(\mathrm{sec} / \mathrm{veh} / \mathrm{mi})\end{array}$ & $\begin{array}{c}\text { Average \# } \\
\text { of stops }\end{array}$ & $\begin{array}{c}\text { Average } \\
\text { Density } \\
(\mathrm{veh} / \mathrm{mi})\end{array}$ & $\begin{array}{c}\text { Average } \\
\text { Harmonic Speed } \\
(\mathrm{mph})\end{array}$ & $\begin{array}{c}\text { Average Stop Time } \\
(\mathrm{sec} / \mathrm{veh} / \mathrm{mi})\end{array}$ & $\begin{array}{c}\text { Average } \\
\text { Speed }(\mathrm{mph})\end{array}$ \\
\hline MAXBAND & $61.35(4.63)$ & 1.14 & 10.35 & $24.53(6.95)$ & $46.90(4.56)$ & $26.82(0.35)$ \\
MULTIBAND $p=1$ & $46.51(4.29)$ & 1.11 & 9.56 & $27.23(6.87)$ & $32.23(4.21)$ & $28.88(0.39)$ \\
MULTIBAND $p=2$ & $46.92(4.33)$ & 1.12 & 9.62 & $27.11(6.59)$ & $32.56(4.33)$ & $28.61(0.42)$ \\
MULTIBAND $p=4$ & $43.73(4.26)$ & 1.05 & 9.32 & $27.72(6.32)$ & $31.51(4.01)$ & $29.20(0.31)$ \\
MULTIBAND $p=0$ & $44.23(4.28)$ & 1.07 & 9.50 & $27.59(6.37)$ & $31.92(4.11)$ & $29.11(0.40)$ \\
AM-BAND $p=1$ & $42.71(3.82)$ & 1.07 & 9.37 & $28.01(6.02)$ & $29.91(3.30)$ & $29.78(0.31)$ \\
AM-BAND $p=2$ & $42.68(3.52)$ & 1.06 & 9.33 & $28.35(5.75)$ & $27.61(3.32)$ & $30.12(0.29)$ \\
AM-BAND $p=4$ & $40.49(3.38)$ & 1.02 & 9.19 & $28.70(5.69)$ & $26.82(3.35)$ & $30.55(0.28)$ \\
AM-BAND $p=0$ & $42.75(3.47)$ & 1.07 & 9.45 & $27.95(5.75)$ & $28.05(3.21)$ & $30.35(0.26)$ \\
\hline
\end{tabular}

Note: numbers in parentheses are standard deviations

Table 5

Simulation results for Case II.

\begin{tabular}{ccccccc}
\hline Method & $\begin{array}{c}\text { Average Delay } \\
(\mathrm{sec} / \mathrm{veh} / \mathrm{mi})\end{array}$ & $\begin{array}{c}\text { Average \# } \\
\text { of stops }\end{array}$ & $\begin{array}{c}\text { Average } \\
\text { Density } \\
(\mathrm{veh} / \mathrm{mi})\end{array}$ & $\begin{array}{c}\text { Average } \\
\text { Harmonic Speed } \\
(\mathrm{mph})\end{array}$ & $\begin{array}{c}\text { Average Stop Time } \\
\text { (sec/veh/mi) }\end{array}$ & $\begin{array}{c}\text { Average } \\
\text { Speed (mph) }\end{array}$ \\
\hline MAXBAND & $64.82(5.65)$ & 1.17 & 7.42 & $27.53(14.25)$ & $55.10(6.04)$ & $32.59(0.56)$ \\
MULTIBAND $p=1$ & $53.02(4.75)$ & 1.04 & 7.32 & $30.10(13.70)$ & $40.87(4.41)$ & $36.35(0.49)$ \\
MULTIBAND $p=2$ & $53.95(5.12)$ & 1.06 & 7.40 & $29.35(13.72)$ & $44.40(4.73)$ & $36.12(0.52)$ \\
MULTIBAND $p=4$ & $56.10(5.85)$ & 1.10 & 7.49 & $29.20(13.75)$ & $45.25(5.31)$ & $35.61(0.60)$ \\
MULTIBAND $p=0$ & $55.03(5.35)$ & 1.07 & 7.45 & $29.12(13.74)$ & $44.72(5.10)$ & $35.83(0.59)$ \\
AM-BAND $p=1$ & $49.44(4.40)$ & 0.92 & 6.67 & $30.99(13.30)$ & $38.15(4.11)$ & $36.70(0.53)$ \\
AM-BAND $p=2$ & $50.44(5.45)$ & 0.94 & 6.75 & $30.73(13.42)$ & $39.04(5.09)$ & $36.59(0.54)$ \\
AM-BAND $p=4$ & $51.32(5.86)$ & 0.98 & 7.17 & $29.35(13.54)$ & $40.22(5.35)$ & $36.23(0.63)$ \\
AM-BAND $p=0$ & $50.92(5.51)$ & 0.96 & 7.09 & $30.25(13.49)$ & $39.89(4.95)$ & $36.41(0.63)$ \\
\hline
\end{tabular}

Note: numbers in parentheses are standard deviations 
Table 6

Comparison of performance in terms of average delay.

\begin{tabular}{|c|c|c|c|c|c|c|}
\hline \multirow{3}{*}{ Method } & \multicolumn{3}{|c|}{ Case I } & \multicolumn{3}{|c|}{ Case II } \\
\hline & \multirow{2}{*}{$\begin{array}{l}\text { Average Delay } \\
\text { (sec/veh/mi) }\end{array}$} & \multicolumn{2}{|c|}{$\%$ Improvement Compared to } & \multirow{2}{*}{$\begin{array}{c}\text { Average Delay } \\
\text { (sec/veh/mi) }\end{array}$} & \multicolumn{2}{|c|}{ \% Improvement Compared to } \\
\hline & & MAXBAND & MULTIBAND & & MAXBAND & MULTIBAND \\
\hline$M A X B A N D$ & 61.35 & -- & -- & 64.82 & -- & -- \\
\hline MULTIBAND $p=1$ & 46.51 & $24.19 \%$ & -- & 53.02 & $18.20 \%$ & -- \\
\hline MULTIBAND $p=2$ & 46.92 & $23.52 \%$ & -- & 53.95 & $16.77 \%$ & -- \\
\hline MULTIBAND $p=4$ & 43.73 & $28.72 \%$ & -- & 56.10 & $13.45 \%$ & -- \\
\hline MULTIBAND $p=0$ & 44.23 & $27.91 \%$ & -- & 55.03 & $15.10 \%$ & -- \\
\hline$A M-B A N D p=1$ & 42.71 & $30.38 \%$ & $8.17 \%$ & 49.44 & $23.73 \%$ & $6.75 \%$ \\
\hline$A M-B A N D p=2$ & 42.68 & $30.43 \%$ & $9.04 \%$ & 50.44 & $22.18 \%$ & $6.51 \%$ \\
\hline$A M-B A N D p=4$ & 40.49 & $34.00 \%$ & $7.41 \%$ & 51.32 & $20.83 \%$ & $8.52 \%$ \\
\hline$A M-B A N D p=0$ & 42.75 & $30.32 \%$ & $3.35 \%$ & 50.92 & $21.44 \%$ & $7.47 \%$ \\
\hline
\end{tabular}

Table 7

Comparison of performance in terms of average number of stops.

\begin{tabular}{|c|c|c|c|c|c|c|}
\hline \multirow{3}{*}{ Method } & \multicolumn{3}{|c|}{ Case I } & \multicolumn{3}{|c|}{ Case II } \\
\hline & \multirow{2}{*}{$\begin{array}{l}\text { Average \# of } \\
\text { Stops }\end{array}$} & \multicolumn{2}{|c|}{ \% Improvement Compared to } & \multirow{2}{*}{$\begin{array}{l}\text { Average \# of } \\
\text { Stops }\end{array}$} & \multicolumn{2}{|c|}{ \% Improvement Compared to } \\
\hline & & MAXBAND & MULTIBAND & & MAXBAND & MULTIBAND \\
\hline$M A X B A N D$ & 1.14 & -- & -- & 1.17 & -- & -- \\
\hline MULTIBAND $p=1$ & 1.11 & $2.63 \%$ & -- & 1.04 & $11.11 \%$ & -- \\
\hline MULTIBAND $p=2$ & 1.12 & $1.75 \%$ & -- & 1.06 & $9.40 \%$ & -- \\
\hline MULTIBAND $p=4$ & 1.05 & $7.89 \%$ & -- & 1.10 & $5.98 \%$ & -- \\
\hline MULTIBAND $p=0$ & 1.07 & $6.14 \%$ & -- & 1.07 & $8.55 \%$ & -- \\
\hline$A M-B A N D p=1$ & 1.07 & $6.14 \%$ & $3.60 \%$ & 0.92 & $21.37 \%$ & $11.54 \%$ \\
\hline$A M-B A N D p=2$ & 1.06 & $7.02 \%$ & $5.36 \%$ & 0.94 & $19.66 \%$ & $11.32 \%$ \\
\hline$A M-B A N D p=4$ & 1.02 & $10.53 \%$ & $2.86 \%$ & 0.98 & $16.24 \%$ & $10.91 \%$ \\
\hline$A M-B A N D p=0$ & 1.07 & $6.14 \%$ & $0.00 \%$ & 0.96 & $17.95 \%$ & $10.28 \%$ \\
\hline
\end{tabular}



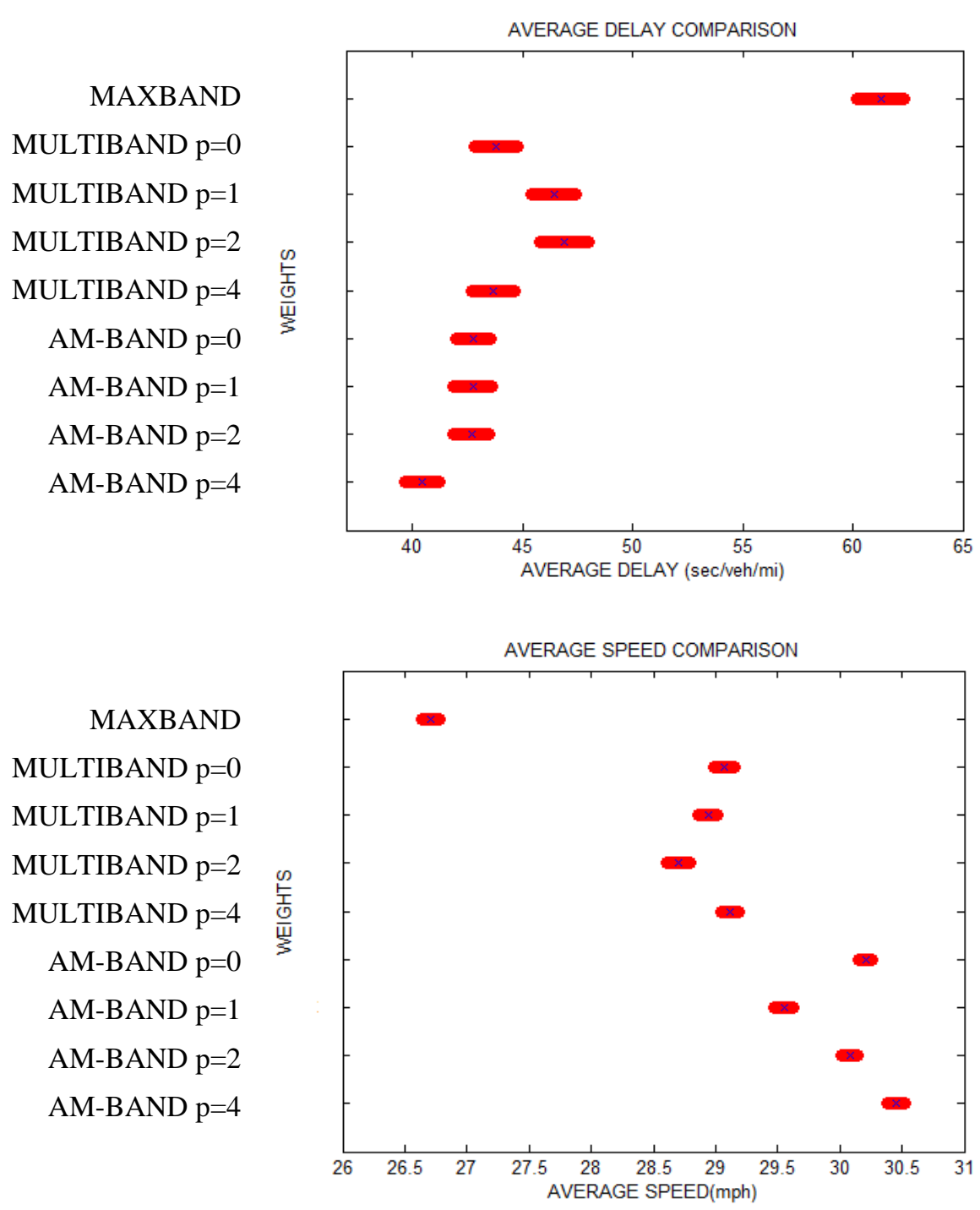

Fig. 6. Distributions of MOEs for case I.

\subsection{Time-space diagrams}

The traffic signal coordination plans generated by MAXBAND, MULTIBAND, and AM-BAND are described in the form of time-space diagrams in Figs. 7 and 8. For MULTIBAND and AM-BAND, their best performing signal coordination plans (i.e., $p=4$ for Case I and $p=1$ for Case II) are presented. For each intersection in these time-space diagrams, the inbound and outbound red intervals for main-street through movements often do not begin or end simultaneously due to reasons such as varying main-street left-turn phase sequences. Therefore, the two red bars (with the top one for outbound and the bottom one for inbound) at each intersection in many cases do not coincide. The lines in Figs. 7 and 8 are the boundaries of progression bands. 

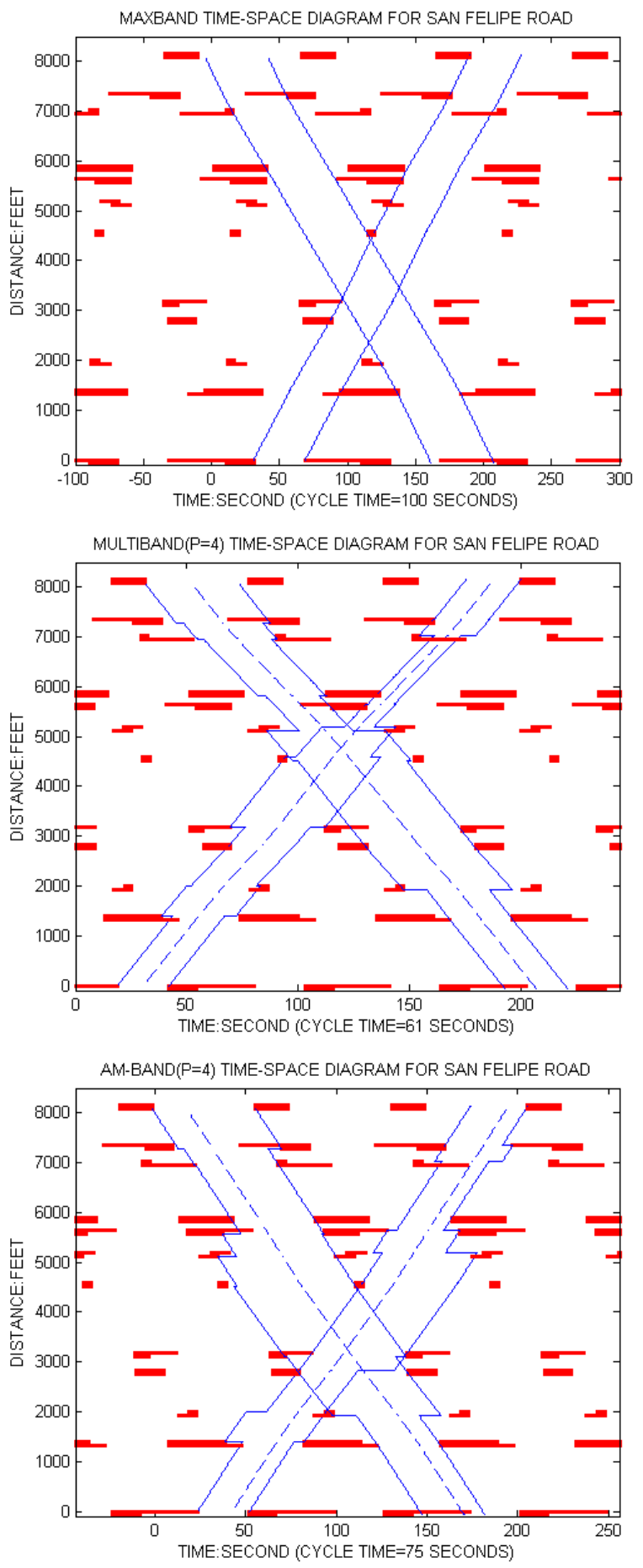

Fig. 7. Time-space diagrams for case I.

Results in Figs. 7 and 8 clearly show the improved flexibility of MULTIBAND and AM-BAND compared to MAXBAND. The MAXBAND model generates two uniform progression bands for the inbound and outbound directions. MULTIBAND generates two progression bands with variable bandwidths that can better accommodate the different segment traffic volumes. Each of the two progression bands is symmetrical with respect to the 
corresponding dashed progression line as shown in Figs. 7 and 8. This symmetry is imposed by the constraints in (6). In AM-BAND, these constraints have been reformulated as discussed at the beginning of Section 3.2 and also shown in (21). The benefits of the reformulated constraints are demonstrated in Figs. 7 and 8. As a result, the two boundaries of the progression bands are not symmetrical with respect to the dashed progression line (and, therefore, cannot be called the centerline anymore). This allows AM-BAND to generate wider bands and to better utilize the available green times for vehicular movements. As shown in Fig. 7, AM-BAND generates a significantly wider band along the outbound direction between 5000 and 7000 feet than MULTIBAND. In Fig. 8, AM-BAND also generates wider bands along the outbound direction between 10000 and 13000 feet and the inbound direction between 1000 and 4000 feet. Due to page limitations, we only include results for $p=4$. For other exponential power $(p)$ values, their corresponding progression bands demonstrate consistent trends as in Figs. 7 and 8. More importantly, these increased bandwidths produce consistent improvements in performance as shown in Tables 5 and 6.
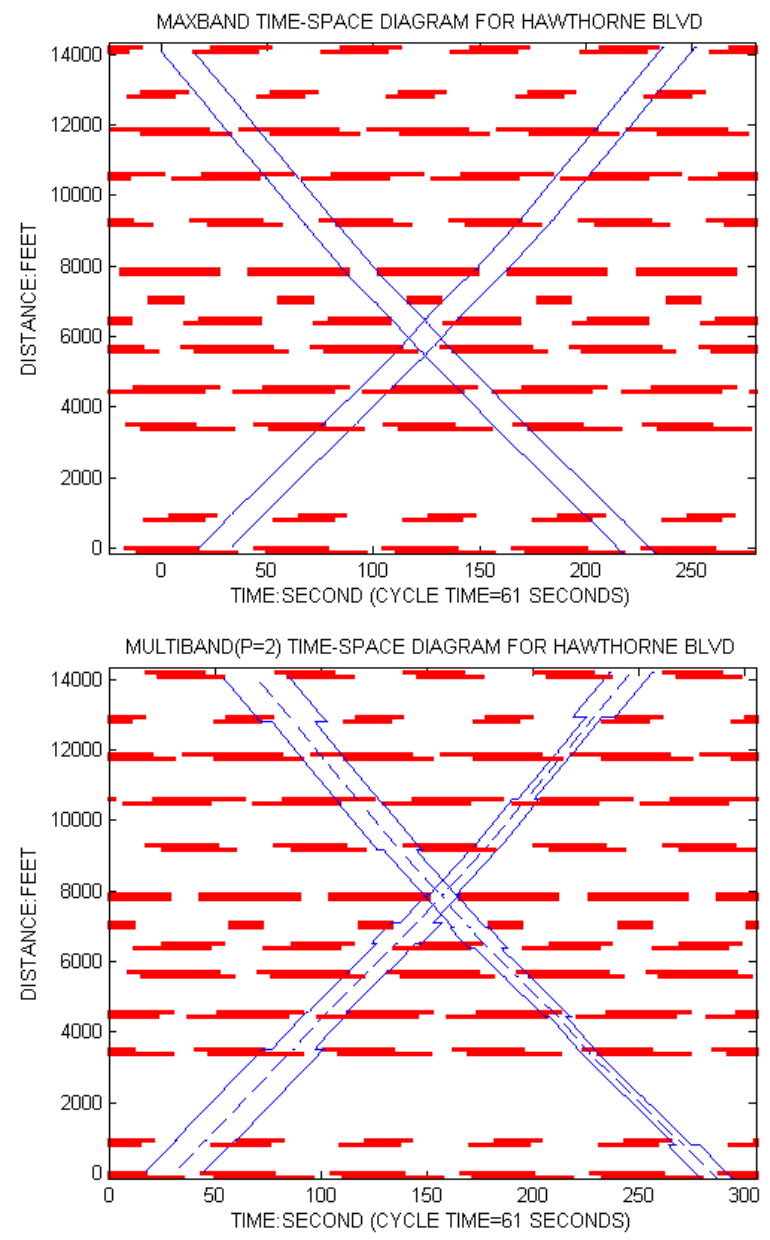


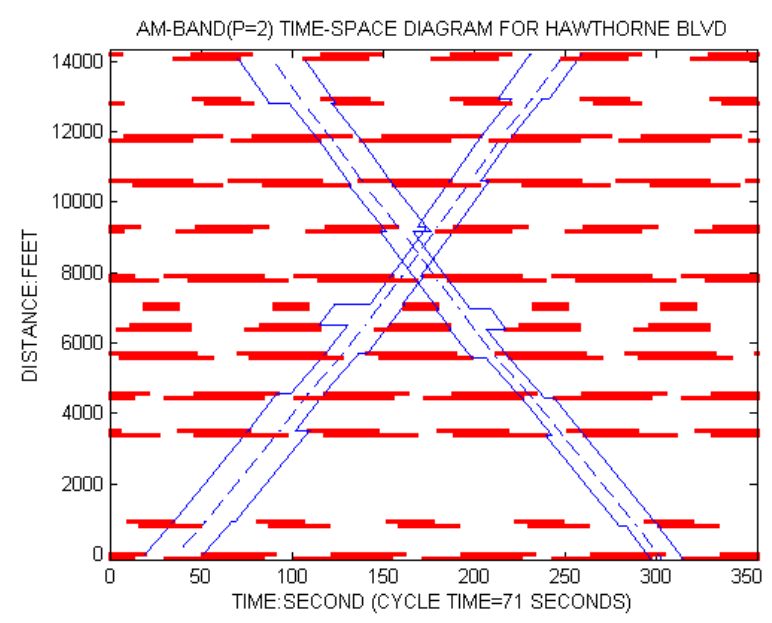

Fig. 8. Time-space diagrams for case II

\section{Sensitivity analysis}

In the proposed AM-BAND model, the bandwidth constraint (22) is used to control how a progression band (e.g., $b_{i}$ ) is separated into two components (e.g., $b^{\prime}{ }_{i}, b^{\prime \prime}{ }_{i}$ ). In particular, this constraint is to prevent one component from being much larger or smaller than the other one, as our empirical results suggest that extremely unbalanced distributions can result in poor model performance. To constrain the ratio between the two components, a bandwidth constraint parameter $q$ is used in (22). By adjusting the value of $q$, one can specify the lower and upper limits of the ratio between the two components of a band. A natural question is how to set the value of $q$ so that the AM-BAND model performance can be optimized. The relationship between the bandwidth constraint parameter and the AM-BAND model performance is complicated and difficult to model mathematically. Therefore, a sensitivity analysis is conducted to identify the best values for $q$. The idea behind the sensitivity analysis is fairly straightforward. First, we tried some evenly spaced numbers in a large range. For this particular study, we found that setting $q=2$ resulted in the best AM-BAND model performance. Also, we found that increasing $q$ beyond 2 or decreasing its value below 2 causes the AM-BAND model performance to deteriorate monotonically. We further narrowed down the search space and experimented with a number of values in the vicinity of promising data points (i.e., 2 in our study). For each $q$ value tested, the corresponding traffic signal coordination plan was generated by AM-BAND model and evaluated using AIMSUN. The MOEs for traffic coordination plans with different $q$ values for Case II are shown in Table 7.

As mentioned previously, the results in Table 7 suggest that the performance of the AM-BAND model varies with different $q$ values. Generally, the AM-BAND model performs the best when the value of $q$ is equal to 2 . Another observation is that the changes in the AM-BAND model performance due to varying $q$ values are not very significant, showing that the AM-BAND model performance is stable when $q$ varies between 1.5 and 4 . However, our sensitivity analysis results (not included in Table 7) also suggest that the AM-BAND model performance can be significantly worse when adopting values outside the range of $[1.5,4]$ for $q$. This fact suggests that the difference between the two components of a bandwidth should be within a reasonable range, in which the AM-BAND model maintains its advantage and performs well. 
Table 8

Comparison of the AM-BAND model performance with different trial $q$ values.

\begin{tabular}{|c|c|c|c|c|c|c|c|c|c|c|}
\hline & & $q=1.5$ & $q=2.0$ & $q=2.03125$ & $q=2.0625$ & $q=2.125$ & $q=2.25$ & $q=2.5$ & $q=3.0$ & $q=4.0$ \\
\hline \multirow{2}{*}{$\begin{array}{c}\text { Average } \\
\text { Delay } \\
\text { (sec/veh/mi) }\end{array}$} & $p=1$ & $53.38(5.32)$ & $49.44(4.4)$ & $49.62(4.78)$ & $49.98(5.73)$ & $50.15(5.11)$ & $54.89(5.95)$ & $52.3(3.99)$ & $52.41(4.76)$ & $53.19(4.55)$ \\
\hline & $p=2$ & $58.71(6.7)$ & $50.44(5.45)$ & $50.06(5.15)$ & $50.49(4.77)$ & $50.34(5.38)$ & $53.48(5.28)$ & $52.18(4.25)$ & $52.2(4.17)$ & $62.82(3.48)$ \\
\hline \multirow{2}{*}{$\begin{array}{c}\text { Average \# of } \\
\text { stops }\end{array}$} & $p=1$ & 1 & 0.92 & 0.93 & 0.93 & 0.92 & 0.98 & 0.95 & 0.95 & 0.95 \\
\hline & $p=2$ & 1.02 & 0.94 & 0.93 & 0.93 & 0.94 & 0.97 & 0.95 & 0.95 & 0.9 \\
\hline \multirow{2}{*}{$\begin{array}{c}\text { Average } \\
\text { Speed (mph) }\end{array}$} & $p=1$ & $36.86(0.57)$ & $36.7(0.53)$ & $36.67(0.59)$ & $36.45(0.68)$ & $36.49(0.61)$ & $36.23(0.57)$ & $36.66(0.44)$ & $36.63(0.42)$ & $36.25(0.38)$ \\
\hline & $p=2$ & $35.75(0.55)$ & $36.59(0.54)$ & $36.64(0.56)$ & $36.18(0.59)$ & $36.42(0.6)$ & $36.17(0.61)$ & $36.56(0.42)$ & $36.61(0.39)$ & $33.67(0.59)$ \\
\hline \multirow{2}{*}{$\begin{array}{l}\text { Density } \\
\text { (veh/mi) }\end{array}$} & $p=1$ & 7.03 & 6.67 & 6.69 & 6.68 & 6.72 & 6.98 & 6.83 & 6.86 & 6.96 \\
\hline & $p=2$ & 7.28 & 6.75 & 6.73 & 6.81 & 6.73 & 6.93 & 6.84 & 6.85 & 7.6 \\
\hline \multirow{2}{*}{$\begin{array}{c}\text { Harmonic } \\
\text { Speed (mph) }\end{array}$} & $p=1$ & $29.98(14.37)$ & 30.99 (13.3) & $30.94(13.31)$ & $30.85(13.15)$ & $30.81(13.23)$ & $29.6(14.01)$ & 30.25 (13.93) & $30.22(13.92)$ & 30.02 (13.68) \\
\hline & $p=2$ & $28.7(14.22)$ & $30.73(13.42)$ & 30.83 (13.39) & $30.72(12.96)$ & 30.75 (13.2) & $29.95(13.65)$ & 30.28 (13.79) & $30.28(13.85)$ & $27.8(12.77)$ \\
\hline \multirow{2}{*}{$\begin{array}{c}\text { Average Stop } \\
\text { Time } \\
\text { (sec/veh/mi) }\end{array}$} & $p=1$ & $41.83(4.96)$ & $38.15(4.11)$ & $38.24(4.44)$ & $38.6(5.3)$ & $38.84(4.73)$ & $43.22(5.53)$ & 40.87 (3.72) & $41(4.48)$ & $41.86(4.28)$ \\
\hline & $p=2$ & $46.73(6.17)$ & $39.04(5.09)$ & 38.73 (4.79) & $38.99(4.43)$ & $38.9(5.02)$ & $41.84(4.9)$ & $40.72(3.99)$ & $40.76(3.91)$ & $51.65(3.15)$ \\
\hline
\end{tabular}




\section{Conclusions and discussions}

In this study, an Asymmetrical Multi-BAND (AM-BAND) optimization model for arterial traffic signal coordination has been presented. The model is based on the well-known MULTIBAND model by relaxing its symmetrical progression band requirement. Both AM-BAND and MULTIBAND are developed based on the multi-band/multi-weight concept incorporating a set of systematic traffic-dependent criteria. The AM-BAND method generates progression bands which are not necessarily symmetrical with respect to the progression line. Due to this fact, AM-BAND is able to expand the progression bands on both sides of the progression lines to better utilize the available green times. This has been shown mathematically in (6) and (21) and at the beginning of Section 3.2. The results in Figs. 7 and 8 also demonstrate the advantages of the AM-BAND model compared to MULTIBAND. Using the result in Fig. 7 as an example, the AM-BAND model generates a significantly wider band along the outbound direction between 5000 and 7000 feet than MULTIBAND, while providing approximately the same bandwidths for the other segments. The expanded bandwidths can help to dissipate the overflow queues from previous cycles, thus leading to better overall performance.

The AM-BAND model is formulated as a mixed-integer linear program and solved by the IBM ILOG CPLEX Optimization studio. Its performance is compared with MAXBAND and MULTIBAND based on two case study arterials using AIMSUN simulations. Different weighting strategies (i.e., $p$ in Eq. (3)) are considered. The optimal solutions are evaluated in terms of average delay, number of stops, average speed, stopped time, etc. The evaluation results consistently show that the overall performance of AM-BAND is better than those of MAXBAND and MULTIBAND.

In the AM-BAND model, a bandwidth constraint (17) is introduced to prevent either one of the two bandwidth components (e.g., $b^{\prime}{ }_{i}$ and $b^{\prime \prime}{ }_{i}$ ) from being zero or extremely small. A sensitivity study is conducted to identify the optimal value for the bandwidth constraint parameter $q$. For the two case study arterials, it is found that when $q$ is in the range of 1.5 and 2, the AM-BAND model tends to perform the best. Additionally, the results in Tables 6 and 7 demonstrate that there is no single $p$ value that can produce the best performance in all cases. This reflects the complexity of arterial traffic signal coordination problem. No two arterials are exactly the same. The optimal $p$ value is affected by many factors such as turning traffic counts, inbound/outbound volume ratios and intersection spacing and, perhaps, can only be determined on a case-by-case basis. Clearly, the bandwidth weights play a critical role in determining the arterial performance. Future studies may be conducted to explore optimal values for the optimization parameters, e.g., $a_{i}, q$, and $p$.

\section{References}

Brooks, W.D., undated. Vehicular traffic control - Designing arterial progressions using a digital computer. Data Processing IBM.

Chang, E.C.P., Cohen, S.L., Liu, C., Chaudhary, N. A., and Messer, C., 1988. MAXBAND-86: program for optimizing left-turn phase sequence in multi-arterial closed networks. Transportation Research Record. 1181: 61-67.

Chaudhary, N.A., and Messer, C.J., 1993. PASSER-IV: a program for optimizing signal timing in grid networks. Transportation Research Record. 1421: 82-93.

Cohen, S.L., and Liu, C.C., 1986. The bandwidth-constrained TRANSYT signal-optimization program. Transportation Research Record. 1057: 1-9. 
Gartner, N.H., Assmann, S.F., Lasaga, F.L., and Hou, D.L., 1990. MULTIBAND - A variable-bandwidth arterial progression scheme. Transportation Research Record. 1287: 212-222.

Gartner, N.H., Assmann, S.F., Lasaga, F.L., and Hou, D.L., 1991. A multi-band approach to arterial traffic signal optimization. Transportation Research B. 25B: 55-74.

Gartner, N.H., Little, J.D., and Gabbay H., 1975. Optimization of traffic signal settings by mixed integer linear programming. Transportation Science. 9: 321-343.

Gartner, N.H. and Stamatiadis, C., 2002. Arterial-based control of traffic flow in urban grid networks. Mathematical and Computer Modeling. 35: 657-671.

Hu, P., Zong, T. and Wu, X., 2011. An improved arterial coordinated control method. The 24th ICTPA Annual Conference \& NACGEA International Symposium on Geo-Trans. S4-003: 1-14.

IBM ILOG CPLEX Optimization Studio, Available online at http://www-01.ibm.com/software/integration/optimization/cplex-optimization-studio/, accessed on May 1, 2013.

Little, J.D., 1966. The synchronization of traffic signals by mixed-integer linear programming. Operations Research. 14: 568-594.

Little, J.D.C., Kelson, M.D., Gartner, N.H., 1981. MAXBAND: A program for setting signals on arterials and triangular networks. Transportation Research Record. 759: 40-46.

Liu, C.C., 1988. Bandwidth-constrained delay optimization for signal systems. ITE Journal. 58(12): 21-26.

Lu, S., Liu, X., and Dai, S., 2008. Revised MAXBAND model for bandwidth optimization of traffic flow dispersion. 2008 ISECS International Colloquium on Computing, Communication, Control, and Management. 85-89.

Messer, C.J., Whitson, R.H., Dudek, C.L., and Romano, E.J., 1973. A variable-sequence multiphase progression optimization program. Highway Research Record. 445: 24-33.

Morgan, J.T. and Little, J.D., 1964. Synchronizing traffic signals for maximal bandwidth. Operations Research. 12: 896-912.

Papola, N., 1992. Bandwidth maximization: split and unsplit solutions. Transportation Research B. 26(5): 341-356.

Park, B., Messer, C.J., and Urbanik, T., 1999. Traffic signal optimization program for oversaturated conditions: genetic algorithm approach. Transportation Research Record. 1683: 133-142.

Park, B., Rouphail, N. M., and Sacks, J, 2001. Assessment of stochastic signal optimization method using microsimulation. Transportation Research Record. 1748: 40-45.

Stamatiadis, C. and Gartner, N.H., 1996. MULTIBAND-96: A program for variable-bandwidth progression optimization of multiarterial traffic networks. Transportation Research Record. 1554: 9-17.

Tian, Z.Z., M., Varun, and H.C., Liu., 2008. Effectiveness of Lead-Lag Phasing on Progression Bandwidth. Transportation Research Record. 2080: 22-27.

TSS, 2013. AIMSUN, Transport Simulation Systems. Available online at http://www.aimsun.com/wp/, accessed on May 1, 2013. 